\title{
Meat Yield from Broiler, Indigenous Naked Neck and Full Feathered Chicken of Bangladesh
}

\author{
M. M. Hossain ${ }^{1}$, M. Nishibori ${ }^{2}$ and M. A. Islam ${ }^{2,3 *}$ \\ ${ }^{1}$ Dept. of Poultry Science, Bangladesh Agricultural University, Mymensingh-2202, Bangladesh \\ ${ }^{2}$ Dept. of Bioresource Science, Graduate School of Biosphere Science, Hiroshima University, Higashi- \\ Hiroshima 739-8528, Japan \\ ${ }^{3}$ Dept. of Animal Science, Bangabandhu Sheikh Mujibur Rahman Agricultural University, Gazipur- \\ 1706, Bangladesh \\ ${ }^{*}$ Corresponding author and Email: aminul_dgvc@yahoo.com
}

Received: 26 April 2012

Accepted: 15 November 2012

\begin{abstract}
Six males and 6 females in each genetic group $(\mathrm{G})$ of broiler $(\mathrm{Br})$, indigenous naked neck (Nana) and full feathered chicken (nana) at set three weight groups (WG); $0.75 \mathrm{~kg}, 1.00 \mathrm{~kg}$ and $1.25 \mathrm{~kg}$ were collected from different local markets and slaughtered. Meat yield traits of slaughtered chickens were recorded to identify a suitable $\mathrm{G}$ at a specific weight by comparing the meat yield of 3 Gs. Broiler had the highest breast, breast: dark meat and heart weight followed by Nana and nana while Nana had the highest gizzard weight followed by nana and Br. Dark, thigh, and giblet weight tended to increase in Nana followed by nana and Br. Male performed better than female for yielding meat except breast: dark meat and abdominal fat which was higher in female than that in male. The highest breast: dark meat of broiler, Nana and nana was observed in $1.00 \mathrm{~kg}, 0.75 \mathrm{~kg}$ and $1.25 \mathrm{~kg} \mathrm{WG}$, respectively. Indigenous chickens showed decreased head and feather weight but increased skin weight with increasing live weight. Notwithstanding, the highest skin weight was observed in Br followed by nana and Nana. The remarkable interaction of $G \times \operatorname{sex}(S), G \times W G$, and $G \times S \times W G$ was observed for breast: dark. The above findings reveal that Nana is comparable to $\mathrm{Br}$, and better than nana for yielding meat. Of the feathering types, broiler is superior to nana for yield of meat. However, more studies are needed to confirm these findings.
\end{abstract}

Keywords: Arbor Acre, full feathered chicken, heterozygous naked neck, meat production, sex, weight group

\section{Introduction}

Poultry meat and eggs from indigenous scavenging chickens are widely available in Bangladesh. Now-a-days, broiler farming is growing to meet the demand for animal protein surpassing the growth of the indigenous chicken rearing industry in semi-scavenging or scavenging systems. Despite tremendous growth of the broiler industry, indigenous scavenging chicken constitute nearly $80 \%$ of the total chicken population (DLS, 1998). Huque (1993) reported that about $78 \%$ of poultry meat comes from free range reared mongrels.

The exotic birds so far introduced in Bangladesh are not well adapted in scavenging systems because of their higher nutritional demand, lower disease resistance and adverse climatic condition. Indigenous chickens are well adapted to the harsh tropical environment, highly resistant to afflatoxin, avian influenza and 
Gumboro diseases but they are susceptible to other important diseases like Newcastle disease, fowl cholera and pox (Branckaert, 2007; Islam and Nishibori, 2009). These diseases may be prevented by using locally produced vaccines. Of the two types of indigenous chicken, indigenous naked neck is considered to be better at heat dissipation and heat tolerance. They are well adapted to tropical climate and more resistant to disease compared to indigenous full feathered counterparts (Merat, 1986; Horst, 1988; Barua and Howlider, 1991; Islam, 2006; Islam and Nishibori, 2009). Indigenous naked neck chicken performs better in terms of growth, meat yield, egg production and survivability compared to its indigenous full feathered counter parts (Barua et al., 1998; Islam and Nishibori, 2009). The diet used in commercial broiler farming is often supplied with antibiotic, toxic binder, hormone and miscellaneous growth promoters which may have harmful effects on humans as well as on poultry (Langhout, 2000).

Broiler carcass contains high fat, less protein and higher cholesterol (Mendes et al., 1994). The meat and egg of indigenous chickens are widely preferred by consumers because of their lean meat (less fat and cholesterol), more protein content, taste, pigmentation and suitability for special dishes which even if they fetch premium prices compared to the products from exotic chickens (Horst, 1991; Islam and Nishibori, 2009).

Therefore, consumers feel no hesitation to pay more money for the products from indigenous chicken (Islam and Nishibori, 2009). Islam and Nishibori (2009) reported that the market price of per $\mathrm{kg}$ live indigenous chicken was almost double of that in broiler. The discrepancy in market price of these two types of chickens is still in increasing trend.

Considering the above facts the present research was undertaken to identify a suitable genetic group of chicken for meat production by comparing meat yield of broiler, indigenous heterozygous naked neck and full feathered chicken at a specific weight.

\section{Materials and Methods}

The experiment was carried out at Bangabandhu Sheikh Mujibur Rahman Agricultural University, (BSMRAU), Gazipur-1706, Bangladesh during the period of May to December 2009.

A total of $36(3 \times 6 \times 2)$ chickens from 3 genetic groups $(G)$ equal number in sex under each $G$ viz.; 12 Arbor Acre commercial broiler hybrids (Br), 12 indigenous heterozygous naked neck (Nana), 12 indigenous full feathered (nana) chickens at 3 weight groups (WG); $0.75 \mathrm{~kg}, 1.00$ $\mathrm{kg}$ and $1.25 \mathrm{~kg}$ were collected from different local markets of Gazipur district. As per consumer demand, only marketable weight of chickens was considered in this study but not age of the birds. Available heterozygous naked neck genotype (Nana) was chosen based on a tuft of feather on the ventral side of the neck region.

The collected birds were fasted for $12 \mathrm{~h}$, and then sacrificed, weighed, eviscerated, dressed, dissected, and the meat stripped from carcass following the method of Jones (1984).

The recorded data of each bird were live weight, weight of feather, head, heart, gizzard, neck, breast meat, thigh meat, drumstick meat, skin, abdominal fat, wing meat, trimmed meat, dark meat (thigh meat + drumstick meat+ wing meat + trimmed meat), total meat (breast meat + dark meat), and weight of thigh bone, drumstick bone, wing bone, and neck weight. Meat yield traits were converted into percentage of individual live weight prior to analyzing the data statistically.

The recorded data for a 3 (genetic group; G) x 2 (sex; S) x 3 (weight group; WG) factorial experiment in Completely Randomized Design were analyzed using Genstat computer package program (Genstat Discovery Edition 1, VSN International Ltd., Hemel Hempstead, HP1 1ES, UK, 2003). Analysis of variance was performed for partitioning the variances to $G, S$, WG, $G \times$ S, G x WG, S x WG, G x S x WG and error to compare meat yield among treatment combinations. Standard error differences (SED) 
were calculated to isolate the significant difference among the mean values.

The following statistical model was used for data analysis.

$\mathrm{Y}_{\mathrm{ijkl}}=\mu+\mathrm{G}_{\mathrm{i}}+\mathrm{S}_{\mathrm{j}}+\mathrm{WG}_{\mathrm{k}}+(\mathrm{G} \times \mathrm{S})_{\mathrm{ij}}+(\mathrm{G} \times$

$\mathrm{WG})_{\mathrm{ik}}+(\mathrm{S} \times \mathrm{WG})_{\mathrm{jk}}+(\mathrm{G} \times \mathrm{S} \times \mathrm{WG})_{\mathrm{ijk}}+\mathrm{e}_{\mathrm{ijkl}}$

Where,

$\mathrm{Y}_{\mathrm{ijk} \mathrm{k}}$ is the observation on $\mathrm{l}^{\text {th }}$ replication of $\mathrm{i}^{\text {th }}$ genetic group, $\mathrm{j}^{\text {th }}$ sex and $\mathrm{k}^{\text {th }}$ weight group.

$\mu$ is the overall mean

$\mathrm{G}_{\mathrm{i}}$ is the fixed effect of $\mathrm{i}^{\text {th }}$ genetic group ( $\mathrm{i}=$ $1,2,3)$

$S_{j}$ is the fixed effect of $j^{\text {th }} \operatorname{sex}(j=1,2)$

$\mathrm{WG}_{\mathrm{k}}$ is the fixed effect of $\mathrm{k}^{\text {th }}$ weight group $(\mathrm{k}=1,2,3)$

$(\mathrm{G} \times \mathrm{S})_{\mathrm{ij}}$ is the interaction effect of $\mathrm{i}^{\text {th }}$ genetic group and $\mathrm{j}^{\text {th }}$ sex

$(\mathrm{G} \times \mathrm{WG}) \mathrm{i}_{\mathrm{k}}$ is the interaction effect of $\mathrm{i}^{\text {th }}$ genetic group and $\mathrm{k}^{\text {th }}$ weight group

$(\mathrm{S} x \mathrm{WG})_{\mathrm{jk}}$ is the interaction effect of $\mathrm{j}^{\text {th }}$ sex and $\mathrm{k}^{\text {th }}$ weight group

$(\mathrm{G} \times \mathrm{S} \times \mathrm{WG})_{\mathrm{ijk}}$ is the interaction effect of $\mathrm{i}^{\text {th }}$ genetic group, $\mathrm{j}^{\text {th }}$ sex and $\mathrm{k}^{\text {th }}$ weight group

$\mathrm{e}_{\mathrm{ijkl}}$ is the random error

\section{Results and Discussion}

The broilers (Br), indigenous naked neck (Nana) and indigenous full feathered (nana) chickens were intended for slaughter at $0.75 \mathrm{~kg}, 1.00 \mathrm{~kg}$ and $1.25 \mathrm{~kg}$ to compare their meat yield traits. But as per availability during purchase from different local markets, $\mathrm{Br}$ chickens were collected and slaughtered at an average weight of $787 \mathrm{~g}$ (835 $\mathrm{g}$ for males; $740 \mathrm{~g}$ for females), $993 \mathrm{~g}$ (1000 $\mathrm{g}$ for males; $985 \mathrm{~g}$ for females) and $1183 \mathrm{~g}$ (1170 $\mathrm{g}$ for males; $1195 \mathrm{~g}$ for females). The Nana chickens were slaughtered at an average weight of $775 \mathrm{~g}$ ( $765 \mathrm{~g}$ for males; $785 \mathrm{~g}$ for females), $1006 \mathrm{~g}$ ( $1037 \mathrm{~g}$ for males; $975 \mathrm{~g}$ for females) and $1273 \mathrm{~g}$ (1395 $\mathrm{g}$ for males and 1150 $\mathrm{g}$ for females). The nana chickens were slaughtered at an average weight of $785 \mathrm{~g}(720 \mathrm{~g}$ for males; $850 \mathrm{~g}$ for females), $1039 \mathrm{~g}$ (1110 $\mathrm{g}$ for males; $968 \mathrm{~g}$ for females) and $1293 \mathrm{~g}(1250 \mathrm{~g}$ for males; $1335 \mathrm{~g}$ for females). The trait(s) which had no significant difference ( $\mathrm{P} \square 0.05$ ) between groups are not shown in tables and text as means.

Breast meat yield and breast: dark meat ratio $(\mathrm{P}<0.01)$, and heart weight $(\mathrm{P}<0.05)$ were the highest in $\mathrm{Br}$, intermediate in Nana and the lowest in nana. But the head weight was the highest in nana, intermediate in Nana and the lowest in $\mathrm{Br}(\mathrm{P}<0.01)$. Gizzard weight was the highest in Nana followed by nana and $\mathrm{Br}$ $(\mathrm{P}<0.01)$, and the highest skin weight was in $\mathrm{Br}$ followed by nana and Nana $(\mathrm{P}<0.01)$. Therefore, of the feathering genetic groups, $\mathrm{Br}$ performed better than that of nana in terms of breast meat, breast: dark meat ratio, heart and skin weight, but nana was better than $\mathrm{Br}$ in terms of head and gizzard weight. Genetic group did not influence the proportion of total meat yield, dark meat, thigh meat, drumstick meat, wing meat, abdominal fat, neck weight, drumstick bone, thigh bone, wing bone, and feather weight. Nevertheless, dark, and thigh weight tended to be increased in Nana followed by nana and $\mathrm{Br}$ (Table 1 and 2).

Genetic group differences were significant for breast meat, breast: dark meat ratio, heart and gizzard weight. The highest breast meat, breast: dark meat ratio and heart weight were in broiler $\mathrm{Br})$ followed by Nana and nana, as corroborated by Islam et al. (2002). They found the highest meat yield in redbro broiler followed by Nana and nana. But the present findings contradicted with the findings of Paul et al. (1990); Hossain et al. (1991); Haque and Howlider (2000). Hossain et al. (1991) found greater breast meat yield in Nana than in broiler. Haque and Howlider (2000) reported the better yield of breast meat, breast: dark meat ratio in indigenous Nana chicken compared to the exotic and their crossbreds. Paul et al. (1990) found the higher breast meat yield in desi (nana) chicken compared to $\mathrm{Br}$. The 
highest gizzard weight was in Nana followed by nana and $\mathrm{Br}$, which coincided with the findings of Paul et al. (1990); Barua and Howlider (1991). The heaviest head was in nana followed by Nana and broiler, may have been affected by age. Nana and perhaps nana were older when they reach different sets of live weight group, and that probably increased their live weight. The other meat yield traits; total meat, dark meat, thigh meat, drumstick meat, neck, drumstick bone, thigh bone, wing bone, and feather weight were almost similar among genetic groups, an observation that disagreed with the findings of Merat (1986); Paul et al. (1990); Haque and Howlider (2000). Paul et al. (1990) reported the higher dressing yield and dark meat in indigenous full feathered chicken (nana) compared to broilers. Haque and Howlider (2000) showed the higher dark, thigh, drumstick meat in indigenous chicken than in broiler. Naked neck had higher dark meat than that of indigenous nana counterparts (Barua and Howlider, 1991). However, Nana was superior to nana in respects of meat yield traits, which consistent with the findings of Hossain et al. (1991); Howlider et al. (1995); Islam and Nishibori (2009).

Irrespective of genetic groups, males had higher yield at $2.54 \%$ more total meat $(\mathrm{P}<0.05), 2.73 \%$ more dark meat $(\mathrm{P}<0.01), 1.45 \%$ more thigh meat $(\mathrm{P}<0.01), 0.97 \%$ more drumstick meat $(\mathrm{P}<0.01), 0.84 \%$ more head weight $(\mathrm{P}<0.01)$, $0.36 \%$ more neck $(\mathrm{P}<0.05), 0.36 \%$ more drumstick bone $(\mathrm{P}<0.05), 0.18 \%$ more thigh bone $(\mathrm{P}<0.05)$ and $0.34 \%$ more wing bone weight, and lower at $0.09 \%$ less breast: dark meat $(\mathrm{P}<0.01)$ and $0.25 \%$ abdominal fat $(\mathrm{P}<0.05)$ compared to female. Sex did not influence $(\mathrm{P} \square 0.05)$ live weight, proportionate breast meat, wing meat, heart, gizzard, skin, and feather weight (Table 1 and 2).

Taking into consideration sex differences, male had a higher proportion of total meat, dark meat, thigh meat, drumstick meat, head, neck, drumstick bone, thigh bone, and wing bone except breast: dark meat ratio and abdominal fat, which were higher in female than that in male, which agreed with the findings of Barua \& Howlider (1991); Howlider et al. (1995); Young et al. (2001); Musa et al. (2006). De et al. (2002) showed the higher thigh and breast meat in female compared to male which contradicted with present findings. Differences between male and female were not observed for the traits of wing meat, breast meat, heart, gizzard, skin, and feather, which disagreed with the findings of Almeida et al. (2000); Abdullah et al. (2010). Almeida et al. (2000) reported the higher wing meat in female than that in male. Abdullah et al. (2010) reported higher breast meat\% but lower leg cut\% in female than that in male chicken at 22 , and 36 days of age. The present study was supported by Dodge and Stadelman (1959) who's showed the similar breast meat yield between male and female chickens.

Relative breast: dark meat ratio of broiler was the highest in $1.00 \mathrm{~kg}$, intermediate in $1.25 \mathrm{~kg}$, and lowest in $0.75 \mathrm{~kg} \mathrm{WG}(\mathrm{P}<0.05)$. But Nana and nana had the highest breast: dark meat ratio in $0.75 \mathrm{~kg}$ and $1.25 \mathrm{~kg}$, and the lowest in $1.00 \mathrm{~kg}$ and $0.75 \mathrm{~kg}$ WG, respectively. Proportionate head and feather weight of indigenous chicken were almost decreased linearly with increasing live weight $(\mathrm{P}<0.01)$. In case of broiler, the highest head and feather weight was in $0.75 \mathrm{~kg}$ and $1.00 \mathrm{~kg}$, and the lowest in $1.00 \mathrm{~kg}$ and 1.25 $\mathrm{kg} \mathrm{WG}$, respectively. Skin weight of indigenous chickens was increased with increasing live weight $(\mathrm{P}<0.05)$. Live weight group did not influence on total meat, breast meat, dark meat, thigh meat, drumstick meat, wing meat, abdominal fat, heart, neck, gizzard, drumstick bone, thigh bone, and wing bone weight.

Live weight group did not affect meat yield traits except breast: dark meat ratio, head, skin and feather weight inconsistent with the observation of Rao and Pillai (1986). The $1.00 \mathrm{~kg} \mathrm{WG}$ of broiler was superior to other WG for breast: dark meat ratio, agreed with Hossain et al. (1991). They found the highest ratio of breast: dark meat in $1.00 \mathrm{~kg} \mathrm{WG}$ compared to $1.25 \mathrm{~kg}$, and $1.50 \mathrm{~kg}$ WG. 
Table 1. Edible meat yield of male $(\mathrm{M})$ and female $(\mathrm{F})$ broilers $(\mathrm{Br})$, indigenous naked neck (Nana) and full feathered (nana) chicken at 3 different live weight groups

\begin{tabular}{|c|c|c|c|c|c|c|c|c|c|c|c|c|c|}
\hline \multirow{2}{*}{ Variable } & \multirow{2}{*}{$\begin{array}{l}\text { Genetic } \\
\text { group }(\mathrm{G})\end{array}$} & \multirow{2}{*}{$\begin{array}{r}\text { Sex } \\
(\mathrm{S})\end{array}$} & \multicolumn{4}{|c|}{ Weight group (WG) } & \multicolumn{7}{|c|}{ SED values and significance level + } \\
\hline & & & 750 & 1000 & 1250 & Mean & $\mathrm{G}$ & $S$ & WG & $\mathrm{G} \times \mathrm{S}$ & GxWG & $\mathrm{S} \times \mathrm{WG}$ & Gx S xWG \\
\hline \multirow{9}{*}{$\begin{array}{l}\text { Live } \\
\text { weight } \\
\text { (g/bird) }\end{array}$} & \multirow[t]{3}{*}{$\mathrm{Br}$} & $\mathrm{M}$ & 835 & 1000 & 1170 & 1002 & $25.10^{\mathrm{NS}}$ & $20.50^{\mathrm{NS}}$ & $25.10^{* * *}$ & $35.60^{\mathrm{NS}}$ & $43.50^{\mathrm{NS}}$ & $35.60^{\mathrm{NS}}$ & $61.60^{* *}$ \\
\hline & & $\mathrm{F}$ & 740 & 985 & 1195 & 973 & & & & & & & \\
\hline & & Mean & 787 & 993 & 1183 & 988 & & & & & & & \\
\hline & \multirow[t]{3}{*}{ Nana } & M & 765 & 1037 & 1395 & 1066 & & & & & & & \\
\hline & & $\mathrm{F}$ & 785 & 975 & 1150 & 970 & & & & & & & \\
\hline & & Mean & 775 & 1006 & 1273 & 1018 & & & & & & & \\
\hline & \multirow[t]{3}{*}{ nana } & M & 720 & 1110 & 1250 & 1027 & & & & & & & \\
\hline & & $\mathrm{F}$ & 850 & 968 & 1335 & 1051 & & & & & & & \\
\hline & & Mean & 785 & 1039 & 1293 & 1039 & & & & & & & \\
\hline \multirow{9}{*}{$\begin{array}{l}\text { Total meat } \\
\text { yield (\%) }\end{array}$} & \multirow[t]{3}{*}{$\mathrm{Br}$} & M & 34.82 & 38.60 & 38.02 & 37.14 & $1.387^{\mathrm{NS}}$ & $1.132^{*}$ & $1.387^{\mathrm{NS}}$ & $1.961^{\mathrm{NS}}$ & $2.402^{\mathrm{NS}}$ & $1.961^{\mathrm{NS}}$ & $3.397^{\mathrm{NS}}$ \\
\hline & & $\mathrm{F}$ & 33.46 & 34.61 & 36.52 & 34.86 & & & & & & & \\
\hline & & Mean & 34.14 & 36.60 & 37.27 & 36.00 & & & & & & & \\
\hline & \multirow[t]{3}{*}{ Nana } & M & 34.39 & 33.53 & 36.78 & 34.90 & & & & & & & \\
\hline & & $\mathrm{F}$ & 34.51 & 31.95 & 32.06 & 32.82 & & & & & & & \\
\hline & & Mean & 34.45 & 32.74 & 34.39 & 33.86 & & & & & & & \\
\hline & \multirow[t]{3}{*}{ nana } & M & 32.22 & 34.77 & 35.03 & 34.01 & & & & & & & \\
\hline & & $\mathrm{F}$ & 27.38 & 32.66 & 32.25 & 30.76 & & & & & & & \\
\hline & & Mean & 29.80 & 33.72 & 33.64 & 32.38 & & & & & & & \\
\hline \multirow{9}{*}{$\begin{array}{l}\text { Breast } \\
\text { meat }(\%)\end{array}$} & \multirow[t]{3}{*}{$\mathrm{Br}$} & M & 15.24 & 20.07 & 18.15 & 17.82 & $0.694^{* * *}$ & $0.567^{\mathrm{NS}}$ & $0.694^{\mathrm{NS}}$ & $0.982^{\mathrm{NS}}$ & $1.203^{\mathrm{NS}}$ & $0.982^{\mathrm{NS}}$ & $1.701^{\mathrm{NS}}$ \\
\hline & & $\mathrm{F}$ & 16.34 & 16.85 & 19.40 & 17.53 & & & & & & & \\
\hline & & Mean & 15.79 & 18.46 & 18.77 & 17.67 & & & & & & & \\
\hline & \multirow[t]{3}{*}{ Nana } & M & 13.96 & 12.85 & 15.13 & 13.98 & & & & & & & \\
\hline & & $\mathrm{F}$ & 15.95 & 14.51 & 14.31 & 14.92 & & & & & & & \\
\hline & & Mean & 14.96 & 13.68 & 14.72 & 14.45 & & & & & & & \\
\hline & \multirow[t]{3}{*}{ nana } & M & 13.11 & 13.48 & 13.87 & 13.48 & & & & & & & \\
\hline & & F & 10.96 & 14.65 & 14.57 & 13.40 & & & & & & & \\
\hline & & Mean & 12.03 & 14.06 & 14.22 & 13.44 & & & & & & & \\
\hline
\end{tabular}




\begin{tabular}{|c|c|c|c|c|c|c|c|c|c|c|c|c|c|}
\hline Contd. & & & & & & & & & & & & & \\
\hline \multirow{9}{*}{$\begin{array}{l}\text { Dark meat } \\
(\%)\end{array}$} & \multirow[t]{3}{*}{$\mathrm{Br}$} & $\mathrm{M}$ & 19.58 & 18.53 & 19.87 & 19.33 & \multirow[t]{9}{*}{$0.864^{\mathrm{NS}}$} & \multirow[t]{9}{*}{$0.705^{* *}$} & \multirow[t]{9}{*}{$0.864^{\mathrm{NS}}$} & \multirow[t]{9}{*}{$1.222^{\mathrm{NS}}$} & \multirow[t]{9}{*}{$1.497^{\mathrm{NS}}$} & \multirow[t]{9}{*}{$1.222^{\mathrm{NS}}$} & \multirow[t]{9}{*}{$2.116^{\mathrm{NS}}$} \\
\hline & & $\mathrm{F}$ & 17.12 & 17.76 & 17.12 & 17.33 & & & & & & & \\
\hline & & Mean & 18.35 & 18.15 & 18.49 & 18.33 & & & & & & & \\
\hline & \multirow[t]{3}{*}{ Nana } & $\mathrm{M}$ & 20.43 & 20.68 & 21.65 & 20.92 & & & & & & & \\
\hline & & $\mathrm{F}$ & 18.55 & 17.44 & 17.69 & 17.90 & & & & & & & \\
\hline & & Mean & 19.49 & 19.06 & 19.67 & 19.41 & & & & & & & \\
\hline & \multirow[t]{3}{*}{ nana } & $\mathrm{M}$ & 19.11 & 21.30 & 21.16 & 20.52 & & & & & & & \\
\hline & & F & 16.41 & 18.00 & 17.68 & 17.37 & & & & & & & \\
\hline & & Mean & 17.76 & 19.65 & 19.42 & 18.94 & & & & & & & \\
\hline \multirow[t]{9}{*}{$\begin{array}{l}\text { Thigh meat } \\
(\%)\end{array}$} & \multirow[t]{3}{*}{$\mathrm{Br}$} & M & 8.84 & 8.17 & 8.58 & 8.53 & \multirow[t]{9}{*}{$0.502^{\mathrm{NS}}$} & \multirow[t]{9}{*}{$0.410^{* * *}$} & \multirow[t]{9}{*}{$0.502^{\mathrm{NS}}$} & \multirow[t]{9}{*}{$0.710^{\mathrm{NS}}$} & \multirow[t]{9}{*}{$0.869^{\mathrm{NS}}$} & \multirow[t]{9}{*}{$0.710^{\mathrm{NS}}$} & \multirow[t]{9}{*}{$1.229^{\mathrm{NS}}$} \\
\hline & & $\mathrm{F}$ & 7.36 & 7.83 & 6.09 & 7.09 & & & & & & & \\
\hline & & Mean & 8.10 & 8.00 & 7.34 & 7.81 & & & & & & & \\
\hline & \multirow[t]{3}{*}{ Nana } & $\mathrm{M}$ & 9.55 & 9.08 & 10.98 & 9.87 & & & & & & & \\
\hline & & $\mathrm{F}$ & 8.86 & 8.32 & 7.74 & 8.31 & & & & & & & \\
\hline & & Mean & 9.21 & 8.70 & 9.36 & 9.09 & & & & & & & \\
\hline & \multirow[t]{3}{*}{ nana } & $\mathrm{M}$ & 8.37 & 9.09 & 10.08 & 9.18 & & & & & & & \\
\hline & & $\mathrm{F}$ & 7.03 & 8.06 & 8.33 & 7.80 & & & & & & & \\
\hline & & Mean & 7.70 & 8.57 & 9.20 & 8.49 & & & & & & & \\
\hline \multirow{9}{*}{$\begin{array}{l}\text { Drumstick } \\
\text { meat }(\%)\end{array}$} & \multirow[t]{3}{*}{$\mathrm{Br}$} & $\mathrm{M}$ & 6.88 & 6.61 & 7.31 & 6.94 & \multirow[t]{9}{*}{$0.300^{\mathrm{NS}}$} & \multirow[t]{9}{*}{$0.245^{* *}$} & \multirow[t]{9}{*}{$0.300^{\mathrm{NS}}$} & \multirow[t]{9}{*}{$0.425^{\mathrm{NS}}$} & $0.520^{\mathrm{NS}}$ & $0.425^{\mathrm{NS}}$ & $0.735^{\mathrm{NS}}$ \\
\hline & & $\mathrm{F}$ & 5.93 & 6.53 & 6.76 & 6.41 & & & & & & & \\
\hline & & Mean & 6.41 & 6.57 & 7.04 & 6.67 & & & & & & & \\
\hline & Nana & M & 7.05 & 7.54 & 7.00 & 7.20 & & & & & & & \\
\hline & & $\mathrm{F}$ & 6.15 & 6.17 & 6.15 & 6.16 & & & & & & & \\
\hline & & Mean & 6.60 & 7.03 & 6.65 & 6.68 & & & & & & & \\
\hline & nana & M & 6.90 & 7.96 & 7.46 & 7.44 & & & & & & & \\
\hline & & $\mathrm{F}$ & 6.29 & 6.11 & 5.84 & 6.08 & & & & & & & \\
\hline & & Mean & 6.60 & 7.03 & 6.65 & 6.76 & & & & & & & \\
\hline
\end{tabular}




\begin{tabular}{|c|c|c|c|c|c|c|c|c|c|c|c|c|c|}
\hline Contd. & & & & & & & & & & & & & \\
\hline \multirow{9}{*}{$\begin{array}{l}\text { Breast: } \\
\text { dark meat }\end{array}$} & \multirow[t]{3}{*}{$\mathrm{Br}$} & M & 0.78 & 0.97 & 0.91 & 0.89 & $0.019^{* *}$ & $0.016^{* *}$ & $0.019^{* * *}$ & $0.027^{* *}$ & $0.033^{*}$ & $0.027^{\mathrm{NS}}$ & $0.047^{*}$ \\
\hline & & $\mathrm{F}$ & 0.85 & 0.95 & 0.87 & 0.89 & & & & & & & \\
\hline & & Mean & 0.81 & 0.96 & 0.89 & 0.89 & & & & & & & \\
\hline & \multirow[t]{3}{*}{ Nana } & M & 0.68 & 0.62 & 0.70 & 0.67 & & & & & & & \\
\hline & & $\mathrm{F}$ & 0.86 & 0.84 & 0.81 & 0.83 & & & & & & & \\
\hline & & Mean & 0.77 & 0.73 & 0.76 & 0.75 & & & & & & & \\
\hline & \multirow[t]{3}{*}{ nana } & M & 0.69 & 0.63 & 0.65 & 0.66 & & & & & & & \\
\hline & & $\mathrm{F}$ & 0.67 & 0.81 & 0.82 & 0.77 & & & & & & & \\
\hline & & Mean & 0.68 & 0.72 & 0.74 & 0.71 & & & & & & & \\
\hline \multirow{9}{*}{ Heart (\%) } & \multirow{3}{*}{$\mathrm{Br}$} & M & 0.60 & 0.53 & 0.53 & 0.55 & $0.034^{*}$ & $0.028^{\mathrm{NS}}$ & $0.034^{\mathrm{NS}}$ & $0.048^{\mathrm{NS}}$ & $0.059^{\mathrm{NS}}$ & $0.048^{\mathrm{NS}}$ & $0.084^{\mathrm{NS}}$ \\
\hline & & $\mathrm{F}$ & 0.62 & 0.44 & 0.56 & 0.54 & & & & & & & \\
\hline & & Mean & 0.61 & 0.48 & 0.54 & 0.54 & & & & & & & \\
\hline & \multirow[t]{3}{*}{ Nana } & M & 0.49 & 0.49 & 0.52 & 0.50 & & & & & & & \\
\hline & & $\mathrm{F}$ & 0.43 & 0.40 & 0.37 & 0.40 & & & & & & & \\
\hline & & Mean & 0.46 & 0.44 & 0.45 & 0.45 & & & & & & & \\
\hline & \multirow[t]{3}{*}{ nana } & M & 0.46 & 0.44 & 0.44 & 0.45 & & & & & & & \\
\hline & & $\mathrm{F}$ & 0.44 & 0.41 & 0.46 & 0.44 & & & & & & & \\
\hline & & Mean & 0.45 & 0.42 & 0.45 & 0.44 & & & & & & & \\
\hline \multirow{9}{*}{$\begin{array}{l}\text { Gizzard } \\
(\%)\end{array}$} & \multirow[t]{3}{*}{$\mathrm{Br}$} & M & 1.52 & 1.39 & 1.13 & 1.35 & $0.189^{* *}$ & $0.155^{\mathrm{NS}}$ & $0.189^{\mathrm{NS}}$ & $0.268^{\mathrm{NS}}$ & $0.328^{\mathrm{NS}}$ & $0.268^{\mathrm{NS}}$ & $0.464^{*}$ \\
\hline & & $\mathrm{F}$ & 1.91 & 1.03 & 1.66 & 1.53 & & & & & & & \\
\hline & & Mean & 1.72 & 1.21 & 1.39 & 1.44 & & & & & & & \\
\hline & \multirow[t]{3}{*}{ Nana } & $\mathrm{M}$ & 3.03 & 3.20 & 2.45 & 2.89 & & & & & & & \\
\hline & & $\mathrm{F}$ & 2.61 & 2.39 & 1.84 & 2.28 & & & & & & & \\
\hline & & Mean & 2.82 & 2.79 & 2.15 & 2.59 & & & & & & & \\
\hline & \multirow[t]{3}{*}{ nana } & M & 2.34 & 2.44 & 3.12 & 2.63 & & & & & & & \\
\hline & & F & 3.18 & 2.77 & 1.53 & 2.50 & & & & & & & \\
\hline & & Mean & 2.76 & 2.60 & 2.33 & 2.56 & & & & & & & \\
\hline
\end{tabular}

$+{ }^{\mathrm{NS}} \mathrm{P}>0.05 ;{ }^{*} \mathrm{P}<0.05 ;{ }^{* *} \mathrm{P}<0.01 ;$ All $\mathrm{SED}^{\mathrm{S}}$ are against 18 error degrees of freedom 
Table 2. Inedible meat yield of male (M) and female (F) broilers (Br), indigenous naked neck (Nana) and full feathered (nana) chicken at 3 different live weight groups

\begin{tabular}{|c|c|c|c|c|c|c|c|c|c|c|c|c|c|}
\hline \multirow[b]{2}{*}{ Variable } & \multirow{2}{*}{$\begin{array}{l}\text { Genetic } \\
\text { group } \\
\text { (G) }\end{array}$} & \multirow{2}{*}{$\begin{array}{r}\text { Sex } \\
(\mathrm{S})\end{array}$} & \multirow[b]{2}{*}{750} & \multicolumn{3}{|c|}{ Weight group (WG) } & \multicolumn{7}{|c|}{ SED values and significance level +} \\
\hline & & & & 1000 & 1250 & Mean & G & $S$ & WG & $\mathrm{G} \times \mathrm{S}$ & G x WG & $\mathrm{S} \times \mathrm{WG}$ & G x S x WG \\
\hline \multirow{9}{*}{$\begin{array}{l}\text { Abdominal } \\
\text { fat }(\%)\end{array}$} & \multirow{2}{*}{$\mathrm{Br}$} & M & 0.22 & 0.37 & 0.59 & 0.39 & $0.088^{\mathrm{NS}}$ & $0.072^{*}$ & $0.088^{\mathrm{NS}}$ & $0.124^{\mathrm{NS}}$ & $0.152^{\mathrm{NS}}$ & $0.124^{\mathrm{NS}}$ & $0.215^{\mathrm{NS}}$ \\
\hline & & $\mathrm{F}$ & 0.30 & 0.48 & 0.73 & 0.50 & & & & & & & \\
\hline & \multirow{3}{*}{ Nana } & Mean & 0.26 & 0.42 & 0.66 & 0.45 & & & & & & & \\
\hline & & M & 0.04 & 0.04 & 0.11 & 0.06 & & & & & & & \\
\hline & & $\mathrm{F}$ & 0.19 & 0.57 & 0.43 & 0.40 & & & & & & & \\
\hline & \multirow{4}{*}{ nana } & Mean & 0.12 & 0.30 & 0.27 & 0.23 & & & & & & & \\
\hline & & M & 0.43 & 0.04 & 0.08 & 0.19 & & & & & & & \\
\hline & & $\mathrm{F}$ & 0.21 & 0.42 & 0.79 & 0.48 & & & & & & & \\
\hline & & Mean & 0.32 & 0.23 & 0.44 & 0.33 & & & & & & & \\
\hline \multirow{9}{*}{$\begin{array}{l}\text { Head weight } \\
(\%)\end{array}$} & \multirow[t]{2}{*}{$\mathrm{Br}$} & M & 3.67 & 2.84 & 3.21 & 3.24 & $0.152^{* *}$ & $0.124^{* *}$ & $0.152^{* *}$ & $0.215^{*}$ & $0.263^{\mathrm{NS}}$ & $0.215^{\mathrm{NS}}$ & $0.372^{\mathrm{NS}}$ \\
\hline & & $\mathrm{F}$ & 3.67 & 2.67 & 2.57 & 2.97 & & & & & & & \\
\hline & \multirow{3}{*}{ Nana } & Mean & 3.67 & 2.75 & 2.89 & 3.10 & & & & & & & \\
\hline & & M & 5.19 & 3.98 & 3.88 & 4.35 & & & & & & & \\
\hline & & $\mathrm{F}$ & 3.43 & 3.01 & 3.14 & 3.19 & & & & & & & \\
\hline & \multirow{4}{*}{ nana } & Mean & 4.31 & 3.50 & 3.51 & 3.77 & & & & & & & \\
\hline & & M & 4.74 & 4.16 & 4.10 & 4.33 & & & & & & & \\
\hline & & $\mathrm{F}$ & 3.60 & 3.40 & 2.74 & 3.25 & & & & & & & \\
\hline & & Mean & 4.17 & 3.78 & 3.42 & 3.79 & & & & & & & \\
\hline \multirow{9}{*}{$\begin{array}{l}\text { Neck weight } \\
(\%)\end{array}$} & \multirow{3}{*}{$\mathrm{Br}$} & M & 2.93 & 2.95 & 2.55 & 2.81 & $0.189^{\mathrm{NS}}$ & $0.154^{*}$ & $0.189^{\mathrm{NS}}$ & $0.267^{*}$ & $0.328^{\mathrm{NS}}$ & $0.267^{\mathrm{NS}}$ & $0.463^{\mathrm{NS}}$ \\
\hline & & F & 3.18 & 3.22 & 2.73 & 3.05 & & & & & & & \\
\hline & & Mean & 3.06 & 3.08 & 2.64 & 2.93 & & & & & & & \\
\hline & \multirow[t]{3}{*}{ Nana } & M & 3.54 & 3.57 & 3.41 & 3.51 & & & & & & & \\
\hline & & $\mathrm{F}$ & 3.13 & 2.88 & 2.38 & 2.79 & & & & & & & \\
\hline & & Mean & 3.33 & 3.22 & 2.90 & 3.15 & & & & & & & \\
\hline & \multirow[t]{3}{*}{ nana } & M & 3.77 & 3.44 & 3.43 & 3.54 & & & & & & & \\
\hline & & $\mathrm{F}$ & 3.27 & 3.03 & 2.49 & 2.93 & & & & & & & \\
\hline & & Mean & 3.52 & 3.23 & 2.96 & 3.24 & & & & & & & \\
\hline
\end{tabular}




\begin{tabular}{|c|c|c|c|c|c|c|c|c|c|c|c|c|c|}
\hline Contd. & & & & & & & & & & & & & \\
\hline \multirow[t]{9}{*}{ Skin (\%) } & \multirow[t]{3}{*}{$\mathrm{Br}$} & M & 6.94 & 6.15 & 6.38 & 6.49 & $0.359^{* *}$ & $0.293^{\mathrm{NS}}$ & $0.359^{*}$ & $0.508^{\mathrm{NS}}$ & $0.622^{\mathrm{NS}}$ & $0.508^{\mathrm{NS}}$ & $0.879^{*}$ \\
\hline & & F & 5.89 & 5.67 & 6.25 & 5.93 & & & & & & & \\
\hline & & Mean & 6.42 & 5.91 & 6.32 & 6.21 & & & & & & & \\
\hline & \multirow[t]{3}{*}{ Nana } & M & 4.77 & 4.60 & 6.50 & 5.29 & & & & & & & \\
\hline & & $\mathrm{F}$ & 4.02 & 5.35 & 4.54 & 4.64 & & & & & & & \\
\hline & & Mean & 4.40 & 4.98 & 5.52 & 4.96 & & & & & & & \\
\hline & \multirow[t]{3}{*}{ nana } & M & 3.99 & 5.67 & 4.87 & 4.84 & & & & & & & \\
\hline & & $\mathrm{F}$ & 4.69 & 5.07 & 7.85 & 5.87 & & & & & & & \\
\hline & & Mean & 4.34 & 5.37 & 6.36 & 5.36 & & & & & & & \\
\hline \multirow{9}{*}{$\begin{array}{l}\text { Drumstick } \\
\text { bone }(\%)\end{array}$} & \multirow{3}{*}{$\mathrm{Br}$} & M & 2.04 & 2.33 & 2.23 & 2.20 & $0.131^{\mathrm{NS}}$ & $0.107^{*}$ & $0.131^{\mathrm{NS}}$ & $0.185^{* *}$ & $0.226^{\mathrm{NS}}$ & $0.185^{*}$ & $0.320^{\mathrm{NS}}$ \\
\hline & & $\mathrm{F}$ & 2.48 & 2.56 & 2.03 & 2.36 & & & & & & & \\
\hline & & Mean & 2.26 & 2.45 & 2.13 & 2.28 & & & & & & & \\
\hline & \multirow[t]{3}{*}{ Nana } & M & 2.75 & 2.39 & 2.28 & 2.47 & & & & & & & \\
\hline & & $\mathrm{F}$ & 1.73 & 1.66 & 1.09 & 1.49 & & & & & & & \\
\hline & & Mean & 2.24 & 2.03 & 1.68 & 1.98 & & & & & & & \\
\hline & \multirow[t]{3}{*}{ nana } & M & 2.26 & 2.29 & 2.58 & 2.38 & & & & & & & \\
\hline & & $\mathrm{F}$ & 2.60 & 2.54 & 1.27 & 2.14 & & & & & & & \\
\hline & & Mean & 2.43 & 2.42 & 1.92 & 2.26 & & & & & & & \\
\hline \multirow{9}{*}{$\begin{array}{l}\text { Thigh bone } \\
\text { (\%) }\end{array}$} & \multirow[t]{3}{*}{$\mathrm{Br}$} & M & 1.43 & 1.44 & 1.79 & 1.55 & $0.095^{\mathrm{NS}}$ & $0.078^{*}$ & $0.095^{\mathrm{NS}}$ & $0.134^{\mathrm{NS}}$ & $0.165^{\mathrm{NS}}$ & $0.134^{*}$ & $0.233^{\mathrm{NS}}$ \\
\hline & & $\mathrm{F}$ & 1.66 & 1.63 & 1.52 & 1.60 & & & & & & & \\
\hline & & Mean & 1.54 & 1.53 & 1.66 & 1.58 & & & & & & & \\
\hline & \multirow[t]{3}{*}{ Nana } & M & 1.74 & 1.58 & 1.64 & 1.65 & & & & & & & \\
\hline & & $\mathrm{F}$ & 1.51 & 1.33 & 1.18 & 1.34 & & & & & & & \\
\hline & & Mean & 1.62 & 1.46 & 1.41 & 1.50 & & & & & & & \\
\hline & \multirow[t]{3}{*}{ nana } & M & 1.52 & 1.82 & 1.90 & 1.74 & & & & & & & \\
\hline & & $\mathrm{F}$ & 1.83 & 1.46 & 1.11 & 1.47 & & & & & & & \\
\hline & & Mean & 1.67 & 1.64 & 1.50 & 1.60 & & & & & & & \\
\hline
\end{tabular}




\begin{tabular}{|c|c|c|c|c|c|c|c|c|c|c|c|c|c|}
\hline Contd. & & & & & & & & & & & & & \\
\hline \multirow{9}{*}{$\begin{array}{l}\text { Wing bone } \\
(\%)\end{array}$} & \multirow[t]{3}{*}{$\mathrm{Br}$} & $\mathrm{M}$ & 2.31 & 1.99 & 2.23 & 2.18 & \multirow[t]{9}{*}{$0.162^{\mathrm{NS}}$} & \multirow[t]{9}{*}{$0.132^{*}$} & \multirow[t]{9}{*}{$0.162^{\mathrm{NS}}$} & \multirow[t]{9}{*}{$0.229^{\mathrm{NS}}$} & \multirow[t]{9}{*}{$0.280^{\mathrm{NS}}$} & \multirow[t]{9}{*}{$0.229^{\mathrm{NS}}$} & \multirow[t]{9}{*}{$0.396^{\mathrm{NS}}$} \\
\hline & & $\mathrm{F}$ & 2.06 & 2.16 & 2.13 & 2.12 & & & & & & & \\
\hline & & Mean & 2.19 & 2.08 & 2.18 & 2.15 & & & & & & & \\
\hline & \multirow[t]{3}{*}{ Nana } & M & 2.58 & 2.48 & 2.07 & 2.38 & & & & & & & \\
\hline & & $\mathrm{F}$ & 1.91 & 1.77 & 1.76 & 1.81 & & & & & & & \\
\hline & & Mean & 2.24 & 2.13 & 1.92 & 2.09 & & & & & & & \\
\hline & \multirow[t]{3}{*}{ nana } & M & 2.36 & 2.07 & 2.57 & 2.33 & & & & & & & \\
\hline & & $\mathrm{F}$ & 2.70 & 1.69 & 1.37 & 1.92 & & & & & & & \\
\hline & & Mean & 2.53 & 1.88 & 1.97 & 2.13 & & & & & & & \\
\hline \multirow{9}{*}{$\begin{array}{l}\text { Feather } \\
\text { weight }(\%)\end{array}$} & \multirow{3}{*}{$\mathrm{Br}$} & $\mathrm{M}$ & 4.21 & 6.00 & 3.84 & 4.68 & \multirow[t]{9}{*}{$0.407^{\mathrm{NS}}$} & \multirow[t]{9}{*}{$0.332^{\mathrm{NS}}$} & \multirow[t]{9}{*}{$0.407^{* *}$} & \multirow[t]{9}{*}{$0.576^{\mathrm{NS}}$} & \multirow[t]{9}{*}{$0.705^{*}$} & \multirow[t]{9}{*}{$0.576^{\mathrm{NS}}$} & \multirow[t]{9}{*}{$0.997^{\mathrm{NS}}$} \\
\hline & & $\mathrm{F}$ & 5.07 & 5.58 & 4.19 & 4.94 & & & & & & & \\
\hline & & Mean & 4.64 & 5.79 & 4.01 & 4.81 & & & & & & & \\
\hline & \multirow[t]{3}{*}{ Nana } & M & 4.39 & 4.37 & 3.21 & 3.99 & & & & & & & \\
\hline & & $\mathrm{F}$ & 4.79 & 3.08 & 3.03 & 3.64 & & & & & & & \\
\hline & & Mean & 4.59 & 3.72 & 3.12 & 3.81 & & & & & & & \\
\hline & \multirow[t]{3}{*}{ nana } & $\mathrm{M}$ & 7.37 & 2.26 & 2.85 & 4.16 & & & & & & & \\
\hline & & $\mathrm{F}$ & 6.46 & 5.17 & 3.34 & 4.99 & & & & & & & \\
\hline & & Mean & 6.92 & 3.72 & 3.10 & 4.58 & & & & & & & \\
\hline
\end{tabular}

$+{ }^{\mathrm{NS}} \mathrm{P}>0.05 ;{ }^{*} \mathrm{P}<0.05 ;{ }^{* * *} \mathrm{P}<0.01 ;$ All $\mathrm{SED}^{\mathrm{S}}$ are against 18 error degrees of freedom 
The differences of breast: dark meat ratio $(\mathrm{P}<0.01)$, head weight, and neck weight $(\mathrm{P}<0.05)$ among genetic groups were more variable in males than those in females. Female of both indigenous types contain more breast: dark meat ratio than that of male. Head and neck weight for all genetic groups were higher in male than that in female. The variations in the proportion of drumstick bone among genetic groups were more variable in female than that in male $(\mathrm{P}<0.01)$. Male of indigenous chicken had heavier drumstick bone than that of female. The interaction of genetic group and sex did not alter live weight, total meat, breast meat, dark meat, thigh meat, drumstick meat, wing meat, abdominal fat, heart, gizzard, skin, thigh bone, wing bone, and feather weight.

Genetic group interacted with sex for the traits of breast: dark meat ratio, head, neck, and drumstick bone. The similar breast: dark meat was found in male and female broiler. In case of indigenous chickens, female had higher breast: dark meat ratio than that of male, which supported by Barua and Howlider (1991); Howlider et al. (1995). Regardless of genetic groups, head and neck weight were higher in male than that in female chicken. Drumstick bone in case of Nana, and nana was heavier in male than in female, but in broiler, female had higher drumstick bone weight than that of male. No interaction of genetic group $\mathrm{x}$ sex was observed for the other meat yield traits. However, total meat yield tended to increase in male compared to female, which supported by Barua \& Howlider (1991); Howlider et al. (1995). Kralik et al. (1993) showed higher wing meat in female in comparison with male Ross and Arbor Acre broiler, which contradicted with the present study.

The genetic group difference for breast: dark meat ratio was more variable in $1.00 \mathrm{~kg} \mathrm{WG}$ than those in $0.75 \mathrm{~kg}$ and $1.25 \mathrm{~kg} \mathrm{WG}(\mathrm{P}<0.05)$. The genetic group difference for feather weight was the highest in $0.75 \mathrm{~kg}$, intermediate in $1.00 \mathrm{~kg}$, and the lowest in $1.25 \mathrm{~kg} W G(\mathrm{P}<0.05)$. The interaction of $\mathrm{G} \times \mathrm{WG}$ did not affect total meat, breast meat, dark meat, thigh meat, drumstick meat, wing meat, abdominal fat, heart, head, neck, gizzard, skin, drumstick bone, thigh bone, and wing bone weight.

Broiler had the highest breast: dark meat ratio in $1.00 \mathrm{~kg}$ WG, supported by Hossain et al. (1991). But in case of nana and Nana, the greater breast: dark meat ratio was observed in $1.25 \mathrm{~kg}$ and $0.75 \mathrm{~kg} \mathrm{WG}$, respectively, contradicted with the findings of Hossain et al. (1991). They found the greater breast: dark meat ratio in Nana and $1.00 \mathrm{~kg}$ WG. The highest feather weight was observed in $0.75 \mathrm{~kg}$ for Nana and nana while broiler had the highest feather weight in $1.00 \mathrm{~kg}$ WG. There was no interaction of $\mathrm{G} \times \mathrm{WG}$ for other meat yield traits.

Sex difference for drumstick and thigh bone weight in favor of males was the highest variable in $1.25 \mathrm{~kg}$ followed by $1.00 \mathrm{~kg}$, and 0.75 $\mathrm{kg} \mathrm{WG}(\mathrm{P}<0.05)$. Interaction of $\mathrm{S}$ and $\mathrm{WG}$ did not influence on total meat, breast meat, dark meat, thigh meat, drumstick meat, wing meat, breast: dark meat ratio, abdominal fat, heart, head, neck, gizzard, skin, wing bone, and feather weight.

As interaction of $\mathrm{S} \times \mathrm{WG}$, male in all weight groups had heavier drumstick bone than in female, however the highest difference between male and female was found in $1.25 \mathrm{~kg}$ followed by $1.00 \mathrm{~kg}$, and $0.75 \mathrm{~kg}$ WG. There was no interaction of S x WG for other meat yield traits, disagreeing with Howlider et al. (1995). They reported the slightly increasing breast meat \%, dark meat $\%$, and breast: dark meat ratio in both sexes with increasing live weight. Avila et al. (1993) showed the significantly higher dressing percentage $(76.8 \%)$ with increasing body weight in male than that in female of Arbor Acres, Pilch Cobb and Hubbard strains inconsistent with the present findings.

Interaction of $\mathrm{G}, \mathrm{S}$ and $\mathrm{WG}$ were remarkable only for breast: dark meat ratio, gizzard, and skin weight $(\mathrm{P}<0.05)$. However, three way interactions did not alter any other meat yield traits. 
Interaction of $\mathrm{G} \times \mathrm{S} \times \mathrm{WG}$ was variable for the traits of breast: dark meat ratio, gizzard and skin weight. However, male broiler contained greater breast: dark meat ratio in $1.00 \mathrm{~kg}$, while Nana and nana female had a greater breast: dark meat ratio in $0.75 \mathrm{~kg}$ and $1.25 \mathrm{~kg} \mathrm{WG}$, respectively.

\section{Conclusions}

The present findings reveal that broiler yielded breast meat, breast: dark meat ratio, and heart weight that better than in Nana, and nana. However, the highest gizzard weight and a tendency for increasing dark, and thigh weight were found in Nana followed by nana, and Br. The other meat yield traits were almost similar among the genetic groups. Therefore, Nana was comparable to broiler, and better than nana for meat yield traits. Of the two feathering types, broiler performed better than nana for meat yield traits. Male was found to be the superior to female for meat yield traits except abdominal fat and breast: dark meat ratio which was higher in female. The suitable WG of broiler, Nana and nana was $1.00 \mathrm{~kg}, 0.75 \mathrm{~kg}$ and $1.25 \mathrm{~kg}$, respectively for meat yield traits. Therefore, this study suggests doing comprehensive research on meat yield traits of 3 genetic groups.

\section{Acknowledgement}

We are grateful to Japan Society for the Promotion of Science (JSPS), for providing a fellowship to carry out the researches in Japan, and prepare this article.

\section{References}

Abdullah, A. Al-Beitawi, Rjoup, M. M. S., Qudsieh, R. I. and Ishmais, M. A. A. 2010. Growth performance, carcass and meat quality characteristics of different commercial crosses of broiler strains of chicken. Journal of Poultry Science, 47: 1321.

Almeida, A. M. D. E. and Zuber, U. 2000. Effect of a feeding and management system on several characteristics of the carcasses of two strains of "free range" type chickens. Veterinaria-Tecnica, 10: 46-50.

Avila, V. S., Ledur, M. C., Bariono, W., Schmidt, G. S. and Costa, C. N. 1993. Growth and carcass quality in broilers of commercial strains. Poultry Abstract, 20: 280 .

Barua, A., Howlider, M. A. R. and Yoshimura, Y. 1998. Indigenous naked neck fowl of Bangladesh. World's Poult.ry Science Journal, 54: 279-286.

Barua, A. and Howlider, M. A. R. 1991. Meat yield of free range naked neck and full feathered Bangladeshi chicken. Indian Journal of Animal Science, 61: 772-775.

Branckaert, R. D. S. 2007. Avian influenza: the new challenges for family poultry. World's Poultry Science Journal, 63: 129-131.

DLS, 1998. Expansions and Activities. Department of Livestock Services. Dhaka, Bangladesh, $31 \mathrm{p}$.

De, P. N., Pal, R. N. and Biswas, S. 2002. Comparative study on the effect of breed and sex on carcass traits on spent duck meat. Indian Journal of Animal Health, 41: 85-88.

Dodge, J. W. and Stadelman, W. J. 1959. Studies on tenderness evaluation. Poultry Science, 38: 1199-1200.

Haque, M. F. and Howlider, M. A. R. 2000. Growth and meat yield in native naked neck, exotic chicken and their crossbreds; $\mathrm{F}_{2}$ generation. Indian Journal of Animal Science, 70: 501-503.

Horst, P. 1988. Using major gene for feather restriction. Poultry Misset, 4: 8-9.

Horst, P. 1991. Native fowl as a reservoir for genomes and major genes with direct and indirect effects on the adaptability and their potential for tropically oriented breeding plans-a review. Animal Research and Development, 33: 63-79. 
Hossain, M. M., Howlider, M. A. R. and Hossain M. J. 1991. Growth performance and meat yield of naked neck Australorps and broiler chickens in a hot-humid environment. The Bangladesh Veterinarian, 8: 4-7.

Howlider, M. A. R., Begum, F., Islam, M. S. and Wahid, M. A. 1995. Feathering and meat yield of full feathered and Naked Neck indigenous chicken of Bangladesh. Journal of Applied Animal Research, 8: 191-195.

Huque, Q. M. E. 1993. Current status of poultry production and marketing systems in Bangladesh. BLRI, Savar, Dhaka, Bangladesh, Sponsored by Bangladesh Agricultural Research Council (USAID/CHECCHI \& COMPANY CONSULTING, INC).

Islam, M. A. 2006. Comparative egg production and egg quality of indigenous full feathered and naked neck chicken at hot-humid climate. Bangladesh Journal of Animal Science, 35: 99-105.

Islam, M. A. and Nishibori M. 2009. Indigenous naked neck chicken: a valuable genetic resource for Bangladesh. World's Poultry Science Journal, 65: 125-138.

Islam, M. A., Seeland, G., Bulbul, S. M. and Howlider, M. A. R. 2002. Meat yield and cooked meat taste of hybrids from different genetic groups in a hot-humid climate. Indian Journal Animal Research, 36: 35-38.

Jones, R. 1984. A standard method for the dissection of poultry for carcass analysis. Technical note 222. The west of Scotland College, UK.

Kralik, G., Petricevic, A., Malter, Z. and Sencic, D. 1993. Effect of genotypes and sex on the yield and quality of meat in fowls. Poultry Science, 47: 39-47.

Langhout, P. 2000. New additives for broiler chickens. Feed Mixture, 24-27 pp.

Mendes, A. A., Garcia, E. A., Gorzales, E. and Politi, S. 1994. Effect of strain on carcass yield in broilers. Poultry Abstract, 20: 103.

Merat, P. 1986. Potential usefulness of the Na (Naked neck) gene in poultry production. World's Poultry Science Journal, 42: 124142.

Musa, H. H., Chen, G. H., Cheng, J. H., Li, B. C. and Mekki, D. M. 2006. Study on carcass characteristics of chicken breeds raised under the intensive condition. International Journal of Poultry Science, 5: 530-533.

Paul, B. P., Howlider, M. A. R. and Bulbul, S. M. 1990. Comparison of meat yield between free range desi and broiler chicken. Indian Journal of Animal Science, 60: 866868.

Rao, K. S. and Pillai, K. J. 1986. Dressing yields of broilers and country chicken reared under identical conditions. Animal Breeding Abstract, 59: 893.

Young, L. L., Northcutt, J. K., Buhr, R. J., Lyon, C. E. and Ware, G. O. 2001. Effects of age, sex and duration of postmortem aging on percentage yield of parts from broiler chicken carcasses. Poultry Science, 80: 376379. 